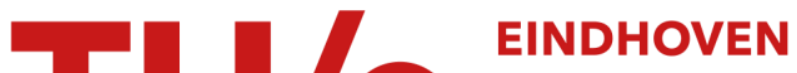 \\ UNIVERSITY OF \\ TECHNOLOGY
}

\section{Petrifying operating guidelines for services}

Citation for published version (APA):

Lohmann, N., \& Wolf, K. (2009). Petrifying operating guidelines for services. In Proceedings 9th International Conference on Application of Concurrency to System Design (ACSD 2009, Augsburg, Germany, July 1-3, 2009)

(pp. 80-88). Institute of Electrical and Electronics Engineers. https://doi.org/10.1109/ACSD.2009.11

DOI:

10.1109/ACSD.2009.11

Document status and date:

Published: 01/01/2009

\section{Document Version:}

Publisher's PDF, also known as Version of Record (includes final page, issue and volume numbers)

\section{Please check the document version of this publication:}

- A submitted manuscript is the version of the article upon submission and before peer-review. There can be important differences between the submitted version and the official published version of record. People interested in the research are advised to contact the author for the final version of the publication, or visit the $\mathrm{DOI}$ to the publisher's website.

- The final author version and the galley proof are versions of the publication after peer review.

- The final published version features the final layout of the paper including the volume, issue and page numbers.

Link to publication

\section{General rights}

Copyright and moral rights for the publications made accessible in the public portal are retained by the authors and/or other copyright owners and it is a condition of accessing publications that users recognise and abide by the legal requirements associated with these rights.

- Users may download and print one copy of any publication from the public portal for the purpose of private study or research.

- You may not further distribute the material or use it for any profit-making activity or commercial gain

- You may freely distribute the URL identifying the publication in the public portal.

If the publication is distributed under the terms of Article 25fa of the Dutch Copyright Act, indicated by the "Taverne" license above, please follow below link for the End User Agreement:

www.tue.nl/taverne

Take down policy

If you believe that this document breaches copyright please contact us at:

openaccess@tue.nl

providing details and we will investigate your claim. 


\title{
Petrifying Operating Guidelines for Services
}

\author{
Niels Lohmann and Karsten Wolf \\ Universität Rostock, Institut für Informatik, 18051 Rostock, Germany \\ \{niels.lohmann, karsten.wolf\}@uni-rostock.de
}

\begin{abstract}
Operating guidelines characterize correct interaction (e.g. deadlock freedom) with a service. They can be stored in a service registry. So far, they have been represented as an annotated transition system.

For the sake of saving space in the registry, we want to translate operating guidelines into Petri nets. To make this possible, we carefully investigate regularities in the annotations.
\end{abstract}

\section{Introduction}

Services (e.g. Web services) are made for loosely coupled interaction [1]. Several aspects are crucial for correct interaction: semantics (compatible interpretation of the meaning of exchanged data), behavior (compatible order of exchanged data), and non-functional (compatible process of exchanging data, including policies, quality of service, etc.).

We contribute to the behavioral aspect of service interaction. In this realm, the concept of operating guidelines [2] plays a key role. An operating guideline $O G_{P}$ of a service $P$ is a finite characterization of the (possibly infinite) set of all those services $R$ that have a compatible behavior w.r.t. $P$. In this paper, we use a compatibility notion that consists of deadlock freedom of the composed system $P \oplus R$ and a given limit for the number of pending messages in a communication channel.

Tasks like checking compatibility can be performed more easily with $O G_{P}$ than with $P$. Such a compatibility check must be performed by a service broker who assigns a fitting (previously published) service $P$ to a query for another service $R$. Other interesting applications of operating guidelines range from checking substitutability (can every user of the old service use the new service, too?) [3], [4] to scenarios of test case generation [5].

Structurally, operating guidelines exploit the fact that the set $\operatorname{Strat}(P)$ of compatible partners (strategies) of a service $P$ contains most permissive ones. A most permissive partner $R^{*}$ can simulate every other partner, i.e. is a top element in the simulation preorder in $\operatorname{Strat}(P)$. To characterize all partners, a most permissive partner is enhanced with Boolean annotations, one for each state. Their propositions correspond to outgoing edges. The formulae control how the behavior may be restricted without destroying compatibility.
Until now, we represented an operating guideline explicitly, as a transition system (of the used most permissive partner). Attempts for symbolic representation using BDD [6] brought some but not sufficient success [7]. It was thus natural to try to transform the transition system into a Petri net, particularly in the face of frequently occurring diamond structures in the transition system. However, the mentioned formulae are attached to states and cannot be naturally assigned to Petri net places or transitions. We introduce the concept of operating guidelines in Sect. 2

In essence, our new approach relies on an implicit representation of the attached formulae. Exploiting regularities in the formulae, we show that they can be reconstructed from two sets of states. This way, the space complexity of an operating guideline is reduced from $|P|\left|R^{*}\right|$ to $\left|R^{*}\right|$ where $|X|$ is the number of states and edges of transition system $X$. This result is presented in Sect. 3 .

In Sect. 4, we show that the matching problem (checking that a given service is represented by a given operating guideline) can be executed using the new representation.

The simpler representation of the attached formulae enables us to condense the state space into a Petri net. For this task, we use existing theory [8], [9] and an existing tool, Petrify [10]. Section 5 briefly explains our approach. In Sect. 6, we discuss the representation of the two sets of states (or markings) in the context of an Petri net representation of a most permissive partner.

We evaluated the Petri net representation of the state space and the new representation of the formulae in a case study. It gives experimental evidence of the significant space reduction that can be obtained and is summarized in Sect. 7. In Sect. 8, we discuss ideas for a further optimization of the Petri net output.

\section{Services and operating guidelines}

In this section, we present the background of our approach.

\subsection{Services}

The behavior of services can be specified in various formalisms, including industrial languages like WS-BPEL [11] or BPMN [12], semiformal languages like UML activity diagrams [13], or formal models like Petri nets, process algebras or state machines (automata). Existing translations 
between WS-BPEL and Petri nets in both directions [14], [15] show that formal models are capable of expressing the relevant behavioral features of services as understood by industry.

We model the behaviour of a service as an automaton. Communication actions (sending or receiving messages) of the service are attached to the transitions. For internal (noncommunicating) transitions, we attach the symbol $\tau$.

Definition 1 (Service automaton). A service automaton $A$ consists of an input alphabet $I$, an output alphabet $O$ such that $I \cap O=\emptyset$ and $\tau \notin(I \cup O)$, a finite set of states $Q$ with an initial state $q_{0} \in Q$ and a set $\Omega \subseteq Q$ of final states, and a transition relation $\delta \subseteq Q \times(I \cup O \cup\{\tau\}) \times Q$ such that $q \in \Omega$ and $\left[q, x, q^{\prime}\right] \in \delta$ implies $x \in I$. We write $q \delta q^{\prime}$ if there is an $x$ such that $\left[q, x, q^{\prime}\right] \in \delta$. Define $\operatorname{en}(q):=\{x \mid$ $\left.\exists q^{\prime}:\left[q, x, q^{\prime}\right] \in \delta\right\}$. A service automaton is deterministic iff, for all $q, x, q^{\prime}, q^{\prime \prime},\left[q, x, q^{\prime}\right] \in \delta$ and $\left[q, x, q^{\prime \prime}\right] \in \delta$ implies $q^{\prime}=q^{\prime \prime}$. A service automaton is responsive iff, for each state $q$, a state $q^{\prime}$ is reachable which is final or enables a transition with a label other than $\tau$.

In the sequel, we consider only responsive service automata which does not restrict practical applicability as nonresponsive behaviour (non-communicating activities forever) is very unusual for services.

An element $\left[q, x, q^{\prime}\right] \in \delta$ is referred to as a transition leaving $q$, arriving at $q^{\prime}$, and labeled $x$. In contrast to I/O automata [16], we assume an asynchronous model of message passing. Furthermore, messages can overtake each other and are not queued on the receiver as, for instance, in the case of communicating finite-state machines [17]. The semantics of message passing is implicitly defined in the following definition of composition of services. For this purpose, we use the concept of multisets $M: I \cup O \rightarrow \mathbb{N}$. We use list notations for describing multisets (e.g., $[a, a, b]$ is the multiset with $[a, a, b](a)=2,[a, a, b](b)=1$, and $[a, a, b](x)=0$, for all other $x) . a \in M$ is true if $M(a)>0$, and the binary operations + and - are performed elementwise. Denote $\operatorname{Bags}(I \cup O)$ the set of all multisets $M$ over $I$ and $O$. The result of composing two services is a transition system.

Definition 2 (Composition). Service automata $A$ and $B$ are composable iff $I_{A}=O_{B}$ and $I_{B}=O_{A}$. The composed system $A \oplus B$ is a transition system consisting of the set of states $Q_{A} \times \operatorname{Bags}\left(I_{A} \cup O_{A}\right) \times Q_{B}$, the initial state $\left[q_{A},[], q_{B}\right]$, a set of final states $\Omega_{A} \times\left\{[1\} \times \Omega_{B}\right.$ and the following transitions:

- (internal move in $A$ :) $\left[\left[q_{A}, M, q_{B}\right],\left[q_{A}^{\prime}, M, q_{B}\right]\right]$, if $\left[q_{A}, \tau, q_{A}^{\prime}\right] \in \delta_{A}$,

- (internal move in $B:$ ) $\left[\left[q_{A}, M, q_{B}\right],\left[q_{A}, M, q_{B}^{\prime}\right]\right]$, if $\left[q_{B}, \tau, q_{B}^{\prime}\right] \in \delta_{B}$

- (send by $A:$ ) $\left[\left[q_{A}, M, q_{B}\right],\left[q_{A}^{\prime}, M+[c], q_{B}\right]\right]$, if $\left[q_{A}, c, q_{A}^{\prime}\right] \in \delta_{A}$ and $c \in O_{A}$,
- (send by $B:$ ) $\left[\left[q_{A}, M, q_{B}\right],\left[q_{A}, M+[c], q_{B}^{\prime}\right]\right]$, if $\left[q_{B}, c, q_{B}^{\prime}\right] \in \delta_{B}$ and $c \in O_{B}$,

- (receive by $A$ :) $\left[\left[q_{A}, M, q_{B}\right],\left[q_{A}^{\prime}, M-[c], q_{B}\right]\right]$, if $\left[q_{A}, c, q_{A}^{\prime}\right] \in \delta_{A}, c \in M$, and $c \in I_{A}$,

- (receive by $B$ :) $\left[\left[q_{A}, M, q_{B}\right],\left[q_{A}, M-[c], q_{B}^{\prime}\right]\right]$, if $\left[q_{B}, c, q_{B}^{\prime}\right] \in \delta_{B}, c \in M$, and $c \in I_{B}$.

In $A \oplus B$, a non-final state without successors is called a deadlock. The composition of $A$ and $B$ respects $k$-limited communication iff, for all reachable states $\left[q_{A}, M, q_{B}\right]$ and all $x, M(x) \leq k$. A service automaton $B$ is a $k$-strategy of a service automaton $A$ iff $A \oplus B$ has $k$-limited communication and no deadlocks are reachable from the initial state.

For $k$-limited communication, the composition is finite. Throughout the paper, let $k$ be arbitrary and fixed. In practice, the value of $k$ may stem from capacity considerations on the channels, from static analysis of the message transfer, or be chosen just sufficiently large.

Example. Fig. 1 depicts two service automata $\mathrm{S}_{\mathrm{A}}\left(I_{\mathrm{S}_{\mathrm{A}}}=\right.$ $\left.\{\mathrm{x}, \mathrm{y}, \mathrm{z}\}, O_{\mathrm{S}_{\mathrm{A}}}=\{\mathrm{a}, \mathrm{b}, \mathrm{c}\}\right)$ and $\mathrm{S}_{\mathrm{B}}\left(I_{\mathrm{S}_{\mathrm{B}}}=\{\mathrm{a}, \mathrm{b}, \mathrm{c}\}\right.$, $\left.O_{S_{B}}=\{x, y, z\}\right)$. We precede transition names with "!" to denote sending transitions and with "?" to denote receiving transitions. In the composition $\mathrm{S}_{\mathrm{A}} \oplus \mathrm{S}_{\mathrm{B}}$, only states that are reachable from the initial state are depicted.

\subsection{Operating guidelines}

Structurally, an operating guideline $O G_{A}$ of a service automaton $A$ is an annotated service automaton.

Definition 3 (Annotated automaton [18]). An annotated automaton consists of a deterministic service automaton $B$ and an annotation $\Phi$ that assigns a Boolean formula to every state $q \in Q_{B}$. The formula $\Phi(q)$ is built upon $e n(q)$ and an additional proposition final. Given an annotated automaton $[B, \Phi]$, another service automaton $C$ with the same alphabet
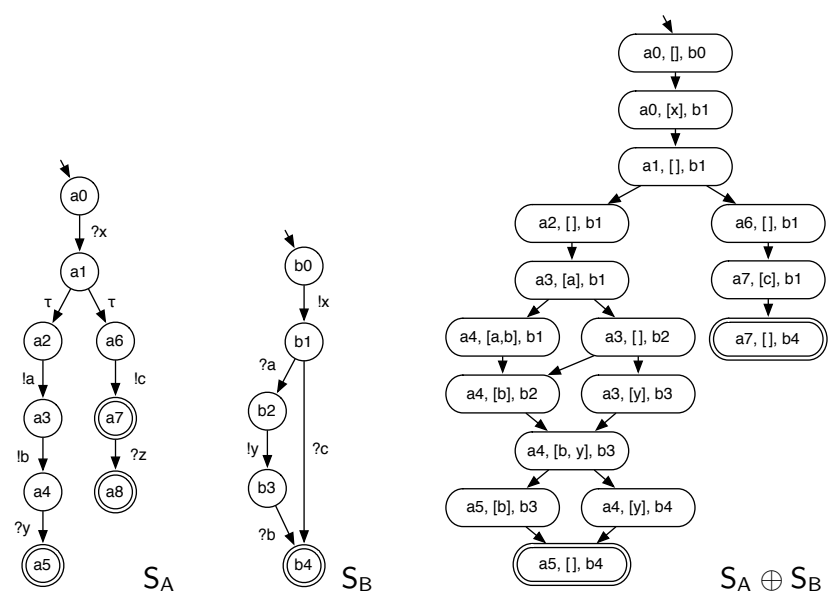

Figure 1: Two service automata and their composition. 
as $B$, a state $q_{B} \in Q_{B}$, and a state $q_{C} \in Q_{C}$, we say that $q_{C}$ models $\Phi\left(q_{B}\right)$ (denoted $q_{C} \models \Phi\left(q_{B}\right)$ ) iff $\Phi\left(q_{B}\right)$ evaluates to true in the following assignment $\beta$ to the propositions. Let $\beta(f i n a l)$ be true iff $q_{C} \in \Omega_{C}$. For other propositions $x$, let $\beta(x)$ be true iff $x \in e n\left(q_{C}\right)$.

According to the definition, annotations to states define requirements about the presence of outgoing edges and the status of a state as final state.

Definition 4 (Matching). A matching between two service automata $A$ and $B$ is a relation $\rho_{A B} \subseteq Q_{A} \times Q_{B}$, inductively defined as follows:

- $q_{0 A} \rho_{A B} q_{0 B}$,

- If $q_{A} \rho_{A B} q_{B}$ and $\left[q_{A}, \tau, q_{A}^{\prime}\right] \in \delta_{A}$ then $q_{A}^{\prime} \rho_{A B} q_{B}$,

- If $q_{A} \rho_{A B} q_{B},\left[q_{A}, x, q_{A}^{\prime}\right] \in \delta_{A},\left[q_{B}, x, q_{B}^{\prime}\right] \in \delta_{B}$, and $x \neq \tau$, then $q_{A}^{\prime} \rho_{A B} q_{B}^{\prime}$.

A matching $\rho_{A B}$ is complete if, for all $q_{A}, q_{A}^{\prime} \in Q_{A}, q_{B} \in$ $Q_{B}$ and $x \neq \tau, q_{A} \rho_{A B} q_{B}$ and $\left[q_{A}, x, q_{A}^{\prime}\right] \in \delta_{A}$ implies that there is an $q_{B}^{\prime}$ such that $\left[q_{B}, x, q_{B}^{\prime}\right] \in \delta_{B}$.

A complete matching $\rho$ is actually a particular weak simulation relation. We disregarded $\tau$-steps in $B$ as we will use matching relations only for $\tau$-free automata $B$ as the following definition suggests.

Definition 5 (Operating guideline). A deterministic $\tau$-free annotated automaton $[B, \Phi]$ is an $k$-operating guideline for a service automaton $A$ iff the following statement is true: $C$ is a $k$-strategy of $A$ if and only if there is a complete matching $\rho_{C B}$ between $C$ and $B$ such that for all $q_{B} \in Q_{B}$ and $q_{C} \in Q_{C}, q_{C} \rho_{C B} q_{B}$ implies $q_{C} \models \Phi\left(q_{B}\right)$.

Example. The operating guideline $\left[S_{C}, \Phi\right]$ of the service automaton $S_{A}$ (Fig. 1) is depicted in Fig. 2. It is easy to see that there exists a complete matching between $S_{B}$ and $\left[S_{C}, \Phi\right]$.

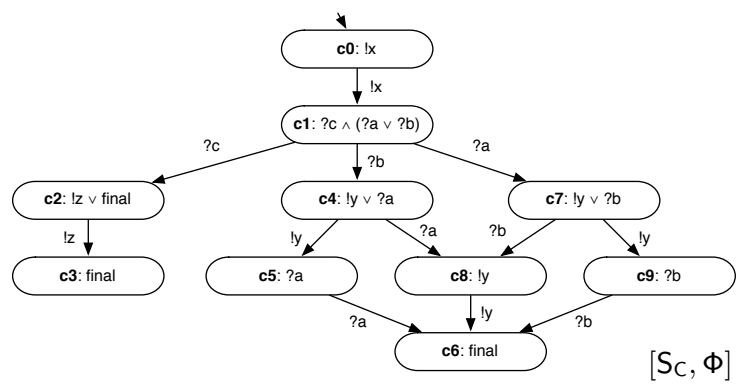

Figure 2: A 1-operating guideline of $\mathrm{S}_{\mathrm{A}}$.

In [2], we proved that every finite state service automaton that has at least one $k$-strategy, has a $k$-operating guideline, too, and presented a construction algorithm that is implemented in the tool Fiona ${ }^{1}$. The proof is based

1. Available for download at http://service-technology.org/fiona. on two observations which we exploit in the sequel. For understanding the results of this article, it is sufficient to consider these observations as granted properties.

The first observation states that the service $B$ underlying the operating guideline $[B, \Phi]$ for $A$ is actually a strategy of $A$.

Proposition 1 ([2]). Let $[B, \Phi]$ be an operating guideline. The identity function $i d$ is a complete matching between $B$ and $[B, \Phi]$ and, for all $q_{B} \in Q_{B}$, $q_{B}=\Phi\left(q_{B}\right)$.

The second observation enlightens the origin of the annotations.

Proposition 2 ([2]). Let $A$ be a service and $[B, \Phi]$ be its operating guideline. Let $C$ be composable with $A$ and assume that $C$ has a complete matching $\rho_{C B}$ with $[B, \Phi]$, the operating guideline for $A$. Then, for a state $q_{C} \in Q_{C}$, there exist $q_{A} \in Q_{A}$ and $M \in \operatorname{Bags}\left(I_{A} \cup O_{A}\right)$ such that $\left[q_{C}, M, q_{A}\right]$ is a reachable deadlock in the composed system $C \oplus A$ if and only if there is a $q_{B} \in Q_{B}$ such that $q_{C} \rho_{C B} q_{B}$ and $q_{C} \not \models \Phi\left(q_{B}\right)$.

In the sequel, we use these observations for deriving our implicit representation of $\Phi$.

\section{Implicit representation of formulae}

In this section, we study a number of regularities in the formulae. These regularities lead us to an alternative representation of operating guidelines.

Throughout this section, we consider an arbitrary operating guideline. We assume that every attached formula $\varphi$ meets the following structural restrictions which do not restrict generality.

- $\varphi$ is represented in conjunctive normal form. i.e. $\varphi=$ $\left\{C_{1}, \ldots, C_{n}\right\}$ for a set of clauses $C_{i}$, where a clause $C_{i}$ is a set of literals (negated or plain propositions).

- $\varphi$ is reduced, i.e. no clause can be replaced by a proper subset, and no clause can be removed, without changing the represented function.

Our first observation says that all send events enabled in $q$ appear positive in every clause.

Lemma 1. Let $A$ be a service, $[B, \Phi]$ its operating guideline, $q \in Q_{B}$, and $x \in O_{B}$. Then the assignment $\beta_{x}$ assigning true to $x$ and false to all other variables satisfies $\Phi(q)$.

Intuition. If a send event $x$ is enabled in state $q_{C}$ of service $C$, then there cannot be a deadlock involving $q_{C}$ as occurrence of $x$ is not restricted. Thus, the sole presence of $x$ must satisfy a corresponding annotation, regardless of other enabled events.

Proof: (Sketch) Remove all transitions from $B$ that leave $q$, except for the transition labeled $x$. Further remove all states and transitions that become unreachable from the 
initial state this way. Let the resulting service be $B^{\prime}$. It can be shown that $i d_{B^{\prime}}$ is a complete matching between $B^{\prime}$ and $B$. As the remaining transition in $q$ is a send transition of $B$, it is enabled in every state $\left[q, M, q_{A}\right]$ of $B^{\prime} \oplus A$. Thus, there cannot be a deadlock of the composed system that involves $q$. By Prop. 2, this means that $q \models \Phi(q)$.

Our second observation is that an operating guideline of a service does never contain negated literals.

Lemma 2. Let $A$ be a service, $[B, \Phi]$ its operating guideline, $q \in Q_{B}$, and $C_{i}$ a clause in $\Phi(q)$. Then $C_{i}$ does not contain negated literals.

Intuition. In a reduced formula, a negated literal corresponds to a non-monotonous formula. Annotations in operating guidelines are, however, intrinsically monotonous as an additional option (another event or turning a non-final state final) can never turn a non-deadlock into a deadlock.

Proof: (Idea) Assume the contrary. Let $C_{i}$ be a clause that contains a negated literal $\neg x$. Since $\Phi(q)$ is assumed to be reduced, there must be an assignment $\beta$ where $\Phi(q)$ yields another value than $\Phi^{\prime}:=\left(\Phi(q) \backslash\left\{C_{i}\right\}\right) \cup\left\{C^{\prime}\right\}$ where $C^{\prime}:=C_{i} \backslash\{\neg x\}$. Clearly, $\beta(x)=$ false, $\beta \models C_{i}, \beta \forall C^{\prime}$ and thus $\beta \models \Phi(q)$. Let $\beta^{\prime}(x)=$ true and $\beta^{\prime}(y)=\beta(y)$, for all $y \neq x$. We have $\beta^{\prime} \not \models C_{i}$ and thus $\beta^{\prime} \not \models \Phi(q)$.

Let first $x \in I_{B} \cup O_{B}$. By Prop. 2, $\beta \models \Phi(q)$ and $\beta^{\prime} \not \models \Phi(q)$ means that there is no deadlock involving $q$ if an outgoing edge labeled $x$ in $q$ is absent while there is a deadlock involving $q$ if such an edge is present. Since an additional edge cannot convert a non-deadlock into a deadlock, this is a contradiction.

Assume second that $x=$ final. However, changing a state from non-final to final can only turn a deadlock into a final state (that is, a non-deadlock), but never a non-deadlock into a deadlock. So again, the occurrence of $\neg$ final contracts the observation in Prop. 2.

The following lemma deals with the appearance of final in $\Phi(q)$.

Lemma 3. Let $A$ be a service, $[B, \Phi]$ its operating guideline, $q \in Q_{B}$, and $C_{i}$ a clause in $\Phi(q)$. If final appears in $C_{i}$ then no elements of $I_{B}$ appear in $C_{i}$.

Intuition. Getting final makes only sense in situations without pending messages as otherwise the composed system would not get into a final state. In a situation without pending messages, however, events in $I_{B}$ are all disabled and cannot help to avoid a deadlock.

Proof: (Sketch) Assume the contrary and consider an assignment $\beta$ to $\Phi(q)$ where all propositions not appearing in $C_{i}$ are set to true and all propositions in $C_{i}$ are set to false. As $\Phi(q)$ is false in this assignment, Prop. 2 states that there is a deadlock $\left[q, M, q_{A}\right]$ in $B^{\prime} \oplus A$ where $B^{\prime}$ is obtained from $B$ by removing transitions labeled $x$ that leave $q$ where $\beta(x)=$ false, and by letting $q$ be a non-final state in $B^{\prime}$. As turning the assignment to final from false to true will let
$\Phi(q)$ evaluate to true, that state must be a final state, that is $q_{A} \in \Omega_{A}$ and $M=\emptyset$. If any element of $I_{B}$ is switched to true instead of final, $\Phi(q)$ evaluates to true again. This would, however, not help to leave the deadlock as $M=\emptyset$. Thus, our assumption contradicts Prop. 2.

In the next two observations, we establish a relation between paths in $B$ and the appearance of elements of $I_{B}$ in clauses of $\Phi(q)$.

Lemma 4. Let $[B, \Phi]$ be the operating guideline of service $A$, $q$ a state of $B, C$ a clause appearing in $\Phi(q)$. If $C$ contains an element $x \in I_{B}$ then there is a path starting in $q$, taking only transitions labeled $x$, and leading to a state $q^{\prime}$ where $\Phi\left(q^{\prime}\right)$ contains a clause $C^{\prime}$ with $C^{\prime} \cap I_{B} \subseteq\left(C \cap I_{B}\right) \backslash\{x\}$.

Intuition. Receiving $x$ by a strategy $C$ can only help escaping a deadlock as long as messages are pending in channel $x$. This is possible at most $k$ times, for the value $k$ fixed throughout this article. Receipt of $k$, however, does not enable any further event in $A$, so the same problem as in $q$ recurs in the $x$ successors of $q$, but ultimately without the opportunity of receiving $x$.

Proof: Let $B^{\prime}$ be obtained from $B$ by removing all edges from $B$ that leave $q$ and have a label that is contained in $C$. As in previous arguments, $i d$ is a complete matching between $B^{\prime}$ and $B$. However, $\Phi(q)$ evaluates to false as all propositions in $C$ are false (by Lemma 3, $C$ cannot contain final as it contains an element of $I_{B}$ ). Thus, $A \oplus B^{\prime}$ contains a deadlock $\left[q_{A}, M, q\right]$. This means that $M(y)=0$ for all $y \notin C$. If any edge with a label $y \in C \cap I_{B}$ is inserted into $B^{\prime}, C$ is satisfied. Moreover, as $\Phi(q)$ is assumed to be reduced, all other clauses are satisfied as well. Consequently, $M(y)>0$, for all $y \in C \cap I_{B}$. Especially we have $M(x)>0$. It is easy to see that receipt of $x$ in $q$ is an activity that is enabled in $B$. Following $x$-transitions $M(x)$ times, we see that some state $\left[q_{A}, M^{\prime}, q^{\prime}\right]$ is reachable in $A \oplus B$ where $M^{\prime}(y)=M(y)$ for $y \neq x$, and $M(x)=0$. In this state, transitions with labels in $I_{B}$ help to prevent a deadlock iff they are contained in $\left(C \cap I_{B}\right) \backslash\{x\}$. Using Lemma 2, $\Phi\left(q^{\prime}\right)$ evaluates to false in every assignment which assigns false to all propositions in $\left(O_{B} \cup\{f i n a l\} \cup C\right) \backslash\{x\}$. Consequently, at least one clause in $\Phi\left(q^{\prime}\right)$ must contain $\left(C \cap I_{B}\right) \backslash\{x\}$. $\square$

For a path $\pi$ in $B$, define $l a b(\pi)$ to be set of labels at transitions taken along $\pi$.

Corollary 1. For every clause $C$ in $\Phi(q)$ there exists a path from $q$ to some state $q^{\prime}$ where $\Phi\left(q^{\prime}\right)$ contains a clause $C^{\prime}$ with $C^{\prime} \cap I_{B}=\emptyset$ and $C \cap I_{B} \supseteq l a b(\pi)$.

The last observation establishes some kind of reversal of Lemma 4.

Lemma 5. Let $x \in I_{B},\left[q, x, q^{\prime}\right]$ be a transition in $B$ and $[B, \Phi]$ the operating guideline of some service $A$. For every $C^{\prime} \in \Phi\left(q^{\prime}\right)$, there is a clause $C \in \Phi(q)$ where $C \cap I_{B} \subseteq$ $\left(C^{\prime} \cap I_{B}\right) \cup\{x\}$. 
Intuition. If receipt of $x$ leads to a situation with certain opportunities to resolve deadlocks, then the same problem is present in the corresponding predecessor state, but now with the additional opportunity of receiving $x$.

Proof: Consider a service $B^{\prime}$ that is obtained from $B$ through inserting a copy $q^{\prime \prime}$ of state $q^{\prime}$. Successors of $q^{\prime \prime}$ are the same as those of $q^{\prime} . q^{\prime \prime}$ has only one predecessor, $q$. $I d \cup\left\{\left[q^{\prime \prime}, q^{\prime}\right]\right\}$ is a complete matching between $B^{\prime}$ and $B$. Consider $q^{\prime \prime}$ and $\Phi\left(q^{\prime}\right)$. As in Lemma 4, we may conclude that there is a state $\left[q_{A}, M, q^{\prime \prime}\right]$ in $A \oplus B^{\prime}$ where $M(y)>0$ for all $y \in C^{\prime} \cap I_{B}$ and $M(y)=0$ for $y \in I_{B} \backslash C^{\prime}$. As $q$ is the only predecessor of $q^{\prime \prime},\left[q_{A}, M+[x], q\right]$ must be reachable as well. Lemma 2 provides that $\Phi(q)$ must be false if all propositions in $O_{B} \cup C^{\prime} \cup\{x\}$ are false. $\Phi(q)$ must thus contain a clause $C$ with $C \cap I_{B} \subseteq\left(C^{\prime} \cap I_{B}\right) \cup\{x\}$.

From the above observations, we may now derive our implicit representation of the attached Boolean formulae. We claim that we can reproduce all formulae from the following two sets of states.

Definition 6 ( $S$ and $F)$. Let $[B, \Phi]$ be the operating guideline of some service $A$.

- $S:=\left\{q \mid q \in Q_{B}, \exists C \in \Phi(q), C \subseteq O_{B}\right\}$.

- $F:=\left\{q \mid q \in Q_{B}, \exists C \in \Phi(q)\right.$, final $\left.\in C\right\}$.

The original formulae can be retrieved from $B, S$, and $F$ as follows.

Theorem 1. Let $[B, \Phi]$ be the operating guideline of some service $A$. Let $S$ and $F$ be as in Def. 6. Then, for all states $q$ of $B$,

$$
\begin{array}{r}
\Phi(q) \equiv\left\{\left(O_{B} \cap e n(q)\right) \cup l a b(\pi) \mid q \stackrel{\pi}{\rightarrow} q^{\prime}, q^{\prime} \in S \cup F,\right. \\
\left.l a b(\pi) \subseteq I_{B}\right\} \cup \mathcal{F}
\end{array}
$$

where $\mathcal{F}:=\left\{\left(O_{B} \cap e n(q)\right) \cup\{\right.$ final $\left.\}\right\}$, if $q \in F$ and $\mathcal{F}=\emptyset$, otherwise.

Note that the constructed set of clauses is not necessarily reduced.

Proof: Let $\Phi$ be the constructed set of clauses. We show first $\Phi(q) \subseteq \Phi$. Let $C \in \Phi(q)$. By Lemma 2, $C$ does not contain negated literals. By Lemma 1, $C \cap O_{B}=$ $O_{B} \cap e n(q)$, as in all constructed clauses. If final $\in C$ then, by Lemma $3, C \cap I_{B}=\emptyset$. Thus, $C=\left(O_{B} \cap e n(q)\right) \cup\{$ final $\}$. Since, in this case, $q \in F, C \in \Phi$. If final $\notin C$ then Cor. 1 asserts that there is a path $\pi$ with $l a b(\pi) \subseteq C \cap I_{B}$. Using this particular path, Lemma 5 states that $\Phi(q)$ contains a clause $C^{\prime}$ with $C^{\prime} \cap I_{B} \subseteq l a b(\pi)$. With Lemma 1, we conclude $C^{\prime} \backslash\{$ final $\} \subseteq C$. As $\Phi(q)$ is reduced, we may conclude $C=C^{\prime}$. Consequently, $C$ is represented in $\Phi$.

Second, we show that $\Phi \subseteq \Phi(q)$. Let $C \in \mathcal{F}$. Then Lemma 1, Lemma 3, and the definition of $F$ assert that $C \in \Phi(q)$. Let finally $C \in\left\{\left(\left(O_{B} \cap e n(q)\right) \cup l a b(\pi) \mid q \stackrel{\pi}{\longrightarrow}\right.\right.$ $\left.q^{\prime}, q^{\prime} \in S \cup F, \operatorname{lab}(\pi) \subseteq I_{B}\right\}$. Assume that, for the path $\pi$ used for constructing $C, \operatorname{lab}(\pi)$ is minimal w.r.t. set inclusion (other paths would lead to redundant clauses and have thus no impact on the semantics of $\Phi)$. Lemma 5 states that $\Phi(q)$ contains a clause $C^{\prime}$ with $C^{\prime} \cap I_{B} \subseteq \operatorname{lab}(\pi)$. For this $C^{\prime}$, Cor. 1 states that there is a path to some state in $S$ using only labels in $C^{\prime} \cap I_{B}$. Since we assumed minimality of $l a b(\pi)$, the set of labels along this path is $l a b(\pi)$. We may conclude $C^{\prime} \cap I_{B}=l a b(\pi)$. Hence, $C \in \Phi(q)$.

The new representation $[B, S, F]$ has a complexity which is linear in $|B|$. In fact, $S$ and $F$ can be represented as two bits attached to each state of $B$. In contrast, the original representation of an operating guideline $[B, \Phi]$ of a service $A$ has a complexity of $\mathcal{O}(|B||A|)$ since the length of a single formula can be linear in the number of states of $A$.

Example. The formulae of the operating guideline $\left[S_{C}, \Phi\right]$ (Fig. 2) can be represented by the sets $S=\{\mathrm{c} 0, \mathrm{c} 8\}$ and $F=\{\mathrm{c} 2, \mathrm{c} 3, \mathrm{c} 6\}$.

\section{Matching with the new representation}

In this section, we sketch a procedure that checks whether a given service $C$ matches an operating guideline in its new representation $[B, S, F]$. In principle, we proceed as suggested in Def. 5. Using a coordinated depth-first search through $B$ and $C$, we create a complete matching $\rho \subseteq$ $Q_{C} \times Q_{B}$. Then, for all $\left[q_{C}, q_{B}\right] \in \rho$, we need to check for satisfaction of $\Phi\left(q_{B}\right)$. The procedure match sketched in Listing 1 implements this check.

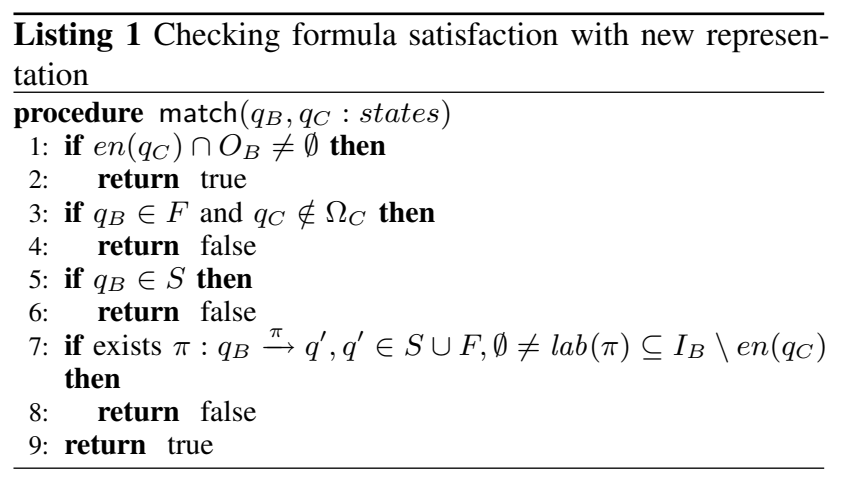

Theorem 2. Procedure match correctly implements the calculation of $\Phi\left(q_{B}\right)$ for an element $\left[q_{C}, q_{B}\right]$ of a complete matching between $B$ and $C$.

Proof: Consider first the case $e n\left(q_{C}\right) \cap O_{B} \neq \emptyset$ (line 1) and let $x \in e n\left(q_{C}\right) \cap O_{B}$. Since $\rho$ is a complete matching, $x \in e n\left(q_{B}\right)$. By Lemma $1, x$ occurs in every clause of $\Phi\left(q_{B}\right)$, so $\Phi\left(q_{B}\right)$ evaluates to true, as returned by match $\left(q_{B}, q_{C}\right)$. Consider next the case $q_{B} \in F$ and $q_{C} \notin \Omega_{C}$ (line 3). As the test of line 1 failed, all propositions in $O_{B}$ take value false. Furthermore, $q_{C} \notin F$ implies that false is assigned final, too. However, with $q_{B} \in F, \Phi\left(q_{B}\right)$ contains a clause $C \subseteq O_{B} \cup\{f i n a l\}$. This clause evaluates to false, so the 
returned value in line 4 is correct. If, in line $5, q_{B} \in S$ then $\Phi\left(q_{B}\right)$ contains a clause $C \subseteq O_{B}$. Since the test in line 1 already failed, this clause evaluates to false and the returned value is correct. In the case specified in the condition of line 5, existence of a nonempty path with labels in $I_{B} \backslash C$ implies existence of a clause $C$ where $C \subseteq O_{B} \cup\left(I_{B} \backslash e n\left(q_{C}\right)\right)$. By the failed test of line 1 , all propositions in $O_{B}$ get value false, and so do all propositions in $I_{B} \backslash e n\left(q_{C}\right)$. Again, the returned value in line 8 is correct. In line 9 , we may conclude the following situation. By the failed test in line 5, there is no clause that contains only propositions in $O_{B}$. Thus, every clause contains final or propositions in $I_{B}$. By the failure of the test in line 3, final does not appear in any clause, or proposition final is true. If a clause contains propositions in $I_{B}$, the failed test in line 7 provides that at least one of them is contained in $e n\left(q_{C}\right)$, too. Since propositions in $e n\left(q_{C}\right)$ get value true, all clauses evaluate to true which justifies the returned value.

The only nontrivial computation in match is the search for a suitable path in line 7 . It can be executed using any kind of graph search algorithm (e.g. depth first search). However, the search occurs only if all tests in lines 1-6 failed. Furthermore, the search space is restricted to transitions with labels in $I_{B} \backslash e n\left(q_{C}\right)$. In addition, we may exploit further structural observations about operating guidelines. As a matter of fact, presence of a path starting in $q$ with labels in $I_{B}$ implies that there is a path for every permutation of the involved labels. Thus, it is sufficient to search only for paths where the sequence of labels is weakly monotonously ascending, for some total order on $I_{B}$. Such a search can be organized by considering transitions only if their label is equal to or greater than the label of the previously taken transition. With all these considerations in mind, procedure match should require only limited computational efforts.

\section{Transforming $B$ into a Petri net}

The automaton $B$ being part of an operating guideline is in fact a labeled transition system. This automaton exhibits a considerable number of so-called diamond structures (cf. Fig. 2), i.e. situations where transitions may occur in any order and lead to the same state. As already mentioned in the previous section, this behavior applies to all sequences of transition with labels in $I_{B}$ but also to all sequences of transitions with labels in $O_{B}$. It is thus reasonable to consider a representation of $B$ as a Petri net.

It is well known that some labeled transition systems can be transformed into a Petri net using a technique known as theory of regions [8], [9]. This technique creates a Petri net with exactly one transition per occurring label in the transition system whenever one exists. In our setting, we cannot assert that $B$ can be represented by a Petri net with just one transition per label. Consequently, we use a generalization of the technique where labels are split into copies of the same label whenever the original technique fails [19]. This technique is intrinsically nondeterministic. So far, we did not explore a domain-specific heuristics for resolving the nondeterminism and just relied on the procedures available in the state-of-the-art tool Petrify [10]. Using this approach, we could, however, achieve a significant reduction in size for the representation of $B$, as the results of the case study (see Sect. 7) suggest.

\section{Representing $S$ and $F$ in a Petri net context}

Assume that the underlying structure $B$ of an operating guideline $[B, S, F]$ is represented as a Petri net. There are two fundamental approaches to the representation of the sets $S$ and $F$ which replace the attached formulae in our approach. We can either represent them explicitly (as a plain enumeration of Petri net markings), or symbolically. A symbolic representation could, for instance, view a set of markings as a set of (Boolean or encoded as Boolean) vectors which can be translated into an implicit representation as a Boolean formula (assigning true to the vectors in the represented set). Approaches of this kind include the Quine/McCluskey algorithm (e.g., see [20]) and the normalization of binary decision diagrams [6]. Despite the availability of these (and many more) advanced techniques, we found that an explicit representation is quite competitive if the following optimizations are met.

We observed that some structural patterns in $B$ give strong indications about presence or absence of states in $S$ or $F$. In particular, we observed:

- a state without successors must be in $F$;

- a state in $F$ is not a member of $S$;

- a state in $S$ is not a member of $F$;

- a state without successors in $I_{B}$ is most likely a member of $S$;

- a state with successors in $I_{B}$ is most likely not a member of $S$.

These observations suggest to explicitly list three sets of states:

Definition $7\left(F^{\prime}, S_{1}, S_{2}\right)$. Let $[B, \Phi]$ be the operating guideline of some service $A$.

$$
\begin{aligned}
& \text { - } F^{\prime}:=F \cap\left\{q \mid q \in Q_{B}, e n(q) \neq \emptyset\right\} . \\
& \text { - } S_{1}:=S \cap\left\{q \mid q \in Q_{B}, e n(q) \cap I_{B} \neq \emptyset\right\} . \\
& \text { - } S_{2}:=\left\{q \mid q \in Q_{B}, e n(q) \cap I_{B}=\emptyset\right\} \backslash(S \cup F) .
\end{aligned}
$$

Corollary 2. The original sets $S$ and $F$ can be reconstructed from the structure of $B$ and the reduced sets $S_{1}, S_{2}$, and $F^{\prime}$ as follows.

$$
\begin{aligned}
& \text { - } F=F^{\prime} \cup\left\{q \mid q \in Q_{B}, e n(q)=\emptyset\right\} . \\
& \text { - } S=S_{1} \cup\left(\left\{q \mid q \in Q_{B}, e n(q) \subseteq O_{B}\right\} \backslash S_{2}\right) \text {. }
\end{aligned}
$$

Without the set $S_{2}$, the set $S$ cannot be correctly reconstructed. The operating guideline $\left[\mathrm{S}_{\mathrm{E}}, \Phi\right]$ (Fig. 3) of the 
service automaton $\mathrm{S}_{\mathrm{D}}$ is an example in which the node e0 has only successors in $O_{E}$ and is not annotated with final. This node's annotation can still be satisfied without setting the literal !v to true. Hence, e $\notin \notin S$. Though the true-annotation characterizes non-responsive services such as $S_{F}$, changing the e0's annotation to "! $\vee \vee$ final" would not help, because this would exclude the responsive service $S_{G}$.

Example. Fig. 4 depicts the result of applying region theory to the structure of the operating guideline of Fig. 2. The formulae are represented by the marking sets $S=\{[\mathrm{p} 0],[\mathrm{p} 4, \mathrm{p} 6]\}$ and $F=\{[\mathrm{p} 5],[\mathrm{p} 6, \mathrm{p} 7],[\mathrm{p} 8]\}$. Exploiting further regularities, these sets can be alternatively represented by $S_{1}=\emptyset, S_{2}=\emptyset$, and $F^{\prime}=\{[\mathrm{p} 5]\}$.

\section{Case Study}

To evaluate the Petri net representation of $B$ and the new representation of the formulae, we applied the presented techniques to several real-life WS-BPEL services from industrial partners. To this end, we translated the WS-BPEL processes into service automata using the tool BPEL2oWFN [14]. Then we used Fiona to calculate the operating guidelines as annotated service automata.

Fiona is able to translate these automata into a Petri net representation using Petrify as backend. We measured the size of $B$ as the sum of its states and edges and the size of $B$ 's Petri net representation as the sum of its places, transitions, and arcs. For the examples, the effect of the reduction dramatically increases with the size of the operating guideline $B$. For the largest examples, the size Petri net representation is less than $1 \%$ of the automaton representation. Table 1 summarizes the results.

Additionally, we implemented the implicit encoding of the nodes' formulae using the sets $S$ and $F$ (Def. 6). The results (see Table 2) show that these sets are-compared to the size of $B$-relatively small. The reduction compares the size of the sets with the number of formulae of $B$. This reduction can be further improved using the sets $S_{1}, S_{2}$, and $F^{\prime}$ (Def. 7): in some cases, no markings had to be stored at all and the largest set contained only 7 markings. Note that for all services of our case studies, the set $S_{2}$ was empty, because situations such as depicted in Fig. 3 are very rare in practice. The data show that the number of elements in the represented sets is such small that a symbolic representation
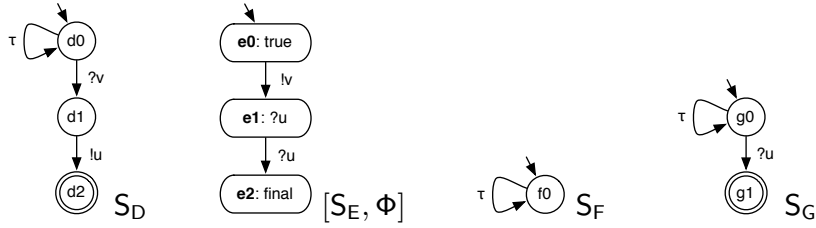

Figure 3: Justification for the set $S_{2}$.

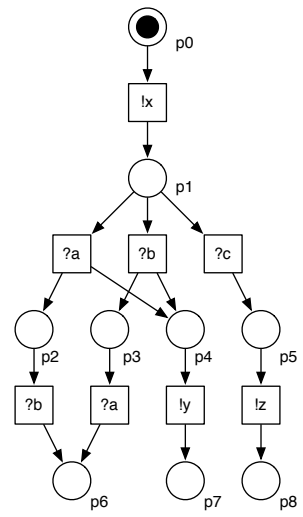

Figure 4: A Petri net representation of $\mathrm{S}_{\mathrm{C}}$.

is not necessary.

The case study of this paper can be replayed using the Web-based implementation of the tool chain available at http://service-technology.org/live/pnog. At the same URL, the tools and the examples of the case study can be downloaded.

\section{Conclusion and future work}

We presented a compact representation of the formulae that are attached to the states of an operating guideline. This representation is not only very space-efficient, but also allows to translate the structure of the operating guideline into a Petri net representation which again is much smaller than the original operating guideline. Experiments show that the

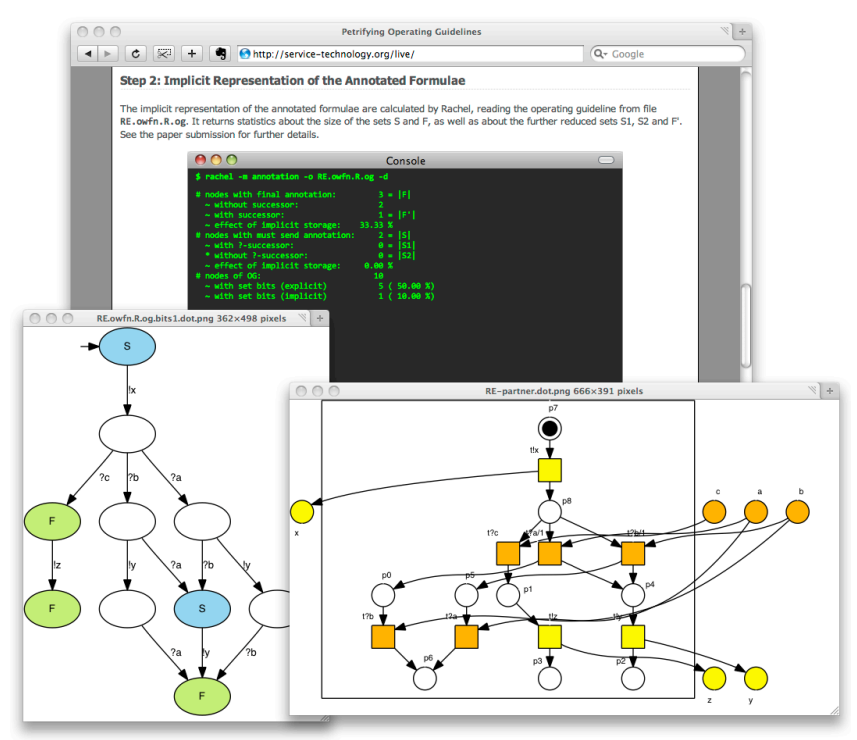

Figure 5: Screen shot of the Web-based implementation available at http://service-technology.org/live/pnog. 
Table 1: Size comparisons between the automaton and the Petri net representation of $B$.

\begin{tabular}{lccc}
\hline service & size $\boldsymbol{B}$ (automaton representation) & size $\boldsymbol{B}$ (Petri net representation) & reduction \\
\hline Ticket Reservation & 403 & 716 & 316 \\
Online Shop & 484 & 249 & $34.72 \%$ \\
Internal Order & 692 & 95 & 76 \\
Travel Service & 776 & $87.76 \%$ \\
Purchase Order & 992 & $92,34 \%$ \\
Reservations & 1866 & $94.96 \%$ \\
Contract Negotiation & 5760 & $97.99 \%$ \\
Deliver Finished Goods & 7792 & 93 & 93 \\
Passport Application & 9472 & $98.81 \%$ \\
Quotation Requisition & 77056 & $99.32 \%$ \\
\hline
\end{tabular}

Table 2: Size comparison between the number of formulae of $B$ and the set representations.

\begin{tabular}{|c|c|c|c|c|c|c|c|c|}
\hline service & formulae of $[B, \Phi]$ & $|S|$ & $|\boldsymbol{F}|$ & reduction & $\left|S_{1}\right|$ & $\left|S_{2}\right|$ & $\left|F^{\prime}\right|$ & reduction \\
\hline Ticket Reservation & 110 & 8 & 2 & $9.09 \%$ & 0 & 0 & 1 & $99.09 \%$ \\
\hline Online Shop & 153 & 11 & 21 & $20.92 \%$ & 0 & 0 & 7 & $95.44 \%$ \\
\hline Internal Order & 184 & 7 & 8 & $8.15 \%$ & 1 & 0 & 4 & $97.28 \%$ \\
\hline Travel Service & 192 & 47 & 2 & $25.52 \%$ & 0 & 0 & 1 & $99.48 \%$ \\
\hline Purchase Order & 232 & 16 & 1 & $7.32 \%$ & 0 & 0 & 0 & $100.00 \%$ \\
\hline Reservations & 369 & 3 & 6 & $2.43 \%$ & 1 & 0 & 5 & $98.37 \%$ \\
\hline Contract Negotiation & 1152 & 26 & 2 & $2.43 \%$ & 0 & 0 & 0 & $100.00 \%$ \\
\hline Deliver Finished Goods & 1376 & 18 & 2 & $1.45 \%$ & 1 & 0 & 1 & $99.85 \%$ \\
\hline Passport Application & 1536 & 3 & 1 & $0.26 \%$ & 0 & 0 & 0 & $100.00 \%$ \\
\hline Quotation Requisition & 11264 & 255 & 1 & $2.27 \%$ & 0 & 0 & 0 & $100.00 \%$ \\
\hline
\end{tabular}

approach is applicable to realistic services.

The size of the synthesized Petri net heavily depends on the level of concurrency in the operating guideline. By adding new states to the operating guideline, the concurrency can be increased and the resulting Petri net might be more compact. To still correctly characterize all strategies of a service, the added nodes then have to be listed in a set similar to the sets introduced in Sect. 6. Likewise, the synthesis algorithm can be adjusted to exploit such domain-specific knowledge. For instance, Carmona et al. [21] present a algorithm without label splitting to apply region theory in the area of process mining [22].

Another interesting direction for future work is the matching between a service automaton and an operating guideline represented by a Petri net. Applying state space reduction techniques such as partial order reduction [23] might help to realize a matching algorithm that avoids to build the complete state space of the operating guideline.

Finally, the concurrency revealed by the region theory might give a deeper insight in the nature of the services characterized by the operating guideline.

\section{Acknowledgment}

N. Lohmann and K. Wolf are supported by the DFG project "Operating Guidelines for Services" (WO 1466/8-1).

\section{References}

[1] K. Gottschalk, "Web Services Architecture Overview," IBM developerWorks, IBM Whitepaper, 2000, http://ibm.com/developerWorks/web/library/w-ovr.

[2] N. Lohmann, P. Massuthe, and K. Wolf, "Operating guidelines for finite-state services," in PETRI NETS 2007, ser. LNCS, vol. 4546. Springer, 2007, pp. 321-341.

[3] W. M. P. v. d. Aalst, N. Lohmann, P. Massuthe, C. Stahl, and K. Wolf, "Multiparty contracts: Agreeing and implementing interorganizational processes," Comput. J., 2009, (In press).

[4] C. Stahl, P. Massuthe, and J. Bretschneider, "Deciding Substitutability of Services with Operating Guidelines," LNCS Transactions on Petri Nets and Other Models of Concurrency, 2009, (In press).

[5] K. Kaschner and N. Lohmann, "Automatic test case generation for interacting services," in ICSOC 2008 Workshops, ser. LNCS. Springer, 2008, (in press).

[6] R. E. Bryant, "Graph-based algorithms for Boolean function manipulation," IEEE Trans. Computers, vol. C-35, no. 8, pp. 677-691, 1986.

[7] K. Kaschner, P. Massuthe, and K. Wolf, "Symbolic representation of operating guidelines for services," Petri Net Newsletter, vol. 72, pp. 21-28, 2007.

[8] A. Ehrenfeucht and G. Rozenberg, "Partial (set) 2-structures. Part I, II," Acta Inf., vol. 27, no. 4, pp. 315-368, 1989. 
[9] E. Badouel, L. Bernardinello, and P. Darondeau, "Polynomial algorithms for the synthesis of bounded nets," in TAPSOFT 1995, ser. LNCS, vol. 915. Springer, 1995, pp. 364-378.

[10] J. Cortadella, M. Kishinevsky, A. Kondratyev, L. Lavagno, and A. Yakovlev, "Petrify: A tool for manipulating concurrent specifications and synthesis of asynchronous controllers," Trans. Inf. and Syst., vol. E80-D, no. 3, pp. 315-325, 1997.

[11] A. Alves et al., "Web Services Business Process Execution Language Version 2.0,” OASIS, OASIS Standard, 2007.

[12] OMG, "Business Process Modeling Notation (BPMN) Version 1.0," OMG, OMG Final Adopted Specification, 2006.

[13] _ _ "Unified Modeling Language (UML)," OMG, Version 2.1.2, 2007

[14] N. Lohmann, "A feature-complete Petri net semantics for WS BPEL 2.0," in WS-FM 2007, ser. LNCS, vol. 4937. Springer, 2008, pp. 77-91.

[15] N. Lohmann and J. Kleine, "Fully-automatic translation of open workflow net models into simple abstract BPEL processes," in Modellierung 2008, ser. LNI, vol. P-127. GI, 2008, pp. 57-72.

[16] N. A. Lynch, Distributed Algorithms. Morgan Kaufmann, 1996.

[17] D. Brand and P. Zafiropulo, "On communicating finite-state machines," J. ACM, vol. 30, no. 2, pp. 323-342, 1983.

[18] A. Wombacher, P. Fankhauser, B. Mahleko, and E. J. Neuhold, "Matchmaking for business processes based on choreographies,' Int. J. Web Service Res., vol. 1, no. 4, pp. 14-32, 2004.

[19] J. Cortadella, M. Kishinevsky, L. Lavagno, and A. Yakovlev, "Deriving Petri nets from finite transition systems," IEEE Trans. Computers, vol. 47, no. 8, pp. 859-882, 1998.

[20] R. F. Tinder, Engineering Digital Design, revised second ed. Academic Press, 2000.

[21] J. Carmona, J. Cortadella, and M. Kishinevsky, "A regionbased algorithm for discovering Petri nets from event logs," in BPM 2008, ser. LNCS, vol. 5240. Springer, 2008, pp. 358-373.

[22] W. M. P. v. d. Aalst, B. v. Dongen, J. Herbst, L. Maruster, G. Schimm, and A. J. M. M. Weijters, "Workflow mining: A survey of issues and approaches," Data Knowl. Eng., vol. 47, no. 2, pp. 237-267, 2003.

[23] A. Valmari, "Stubborn sets for reduced state space generation," in PETRI NETS 1989, ser. LNCS, vol. 483. Springer, 1989, pp. 491-515. 